\section{CONFLICTO, IDENTIDAD Y DESARROLLO EN LA DEMOCRATIZACIÓN DE LAS EX REPÚBLICAS YUGOSLAVAS}

\section{CONFLICT, IDENTITY AND \\ DEVELOPMENT IN THE DEMOCRATIZATION OF THE FORMER YUGOSLAV REPUBLICS}

\author{
Hugo Marcos-Marné \\ Universidad de Salamanca, España. \\ hugomm@usal.es
}

Cómo citar este artículo / Citation: Marcos-Marné, H. 2015. "Conflicto, identidad y desarrollo en la democratización de las ex repúblicas yugoslavas", Revista Internacional de Sociología, 74 (1): e027. Doi: http://dx.doi.org/10.3989/ ris.2016.74.1.027
Copyright: (C) 2016 CSIC. Este es un artículo de acceso abierto distribuido bajo los términos de la licencia Creative Commons Attribution-Non Commercial (by-cn) Spain 3.0.

Recibido: 20/01/2014. Aceptado: 15/10/2014.

Publicación online: 09/02/16

\section{Abstract}

Contrary to the generality of thoughts about political transition in former Yugoslav republics, the observation of ethnic diversity is not able to explain the diversity of results observed in the region by itself. This paper seeks to explain the most important differences in democratization in the 90's decade, using techniques of comparative analysis. To achieve this aim, I am going to analyze elements of identity and development in the different territories, highlighting the essential role of stateness conflict. Deepening in that sense, the practical relevance of these conflicts is reinforced by his contingent nature, which will be developed and justified through empirical results attached to historical arguments.

\section{KEYWORDS}

csQCA; Stateness; Ethnic Fractionalization; Nationalisms; Transitions to Democracy; Yugoslavia. 


\section{INTRODUCCIÓN}

La literatura que trata la cuestión de las transiciones en la región balcánica ha presentado tradicionalmente tres limitaciones que afectan a la extracción de conclusiones. En primer lugar, el enfoque descriptivo predominante en gran parte de los trabajos. En segundo lugar, la ausencia de una aplicación metodológica y de un enfoque comparativo estructurado. En tercer lugar, la explicación del éxito o el fracaso en la transición tomando como causa casi única la inevitabilidad del conflicto violento, a la vista de la diversidad etnocultural (intra e interterritorial). Adicionalmente, en este tipo de estudios la práctica más común ha sido la de tratar los Balcanes de forma general, quedando fuera o menos estudiada la República Socialista Federal Yugoslava (RSFY), siendo este territorio un caso paradigmático de Estado multinacional del que se pueden extraer valiosas conclusiones'.

En línea con lo expuesto encontramos los trabajos de Glenny (1996), Janos (1997), Palacios (2003), Silber y Little (1996), Taibo (1998), Trbovich (2008) o Veiga (1994 y 2011) que poseen una gran riqueza analítica y descriptiva, pero que precisan ser complementados por estudios que utilicen estrategias analíticas comparativas.

Con este objetivo se propone dar respuesta a cuáles son los factores que explican las diferencias en los procesos transicionales, analizando el peso del desarrollo económico, la heterogeneidad étnica y los conflictos de estatalidad. Dicha selección de condiciones busca tener en cuenta los elementos causales más relevantes, y al mismo tiempo centrarse en aquellos que presentan suficientes grados de variación. En concreto, la ausencia del contexto internacional precisa una justificación expresa por el peso que tuvo la diplomacia en el conflicto de los Balcanes. En este sentido hay que señalar la intención de seleccionar variables ligadas a cada uno de los territorios (principalmente condiciones estructurales y comportamiento de elites políticas), siendo prioritario considerar aquellas en las que la variabilidad entre casos es mayor. Adicionalmente el método csQCA exige mantener controlado el número de condiciones en el modelo, para evitar la proliferación de contrafácticos (configuraciones lógicas sin observación empírica), que afectaría sustancialmente a la fiabilidad de las conclusiones obtenidas. Siendo esto así, se propone que el efecto del contexto internacional en las transiciones podría entenderse con mayor profundidad y rigor a través de estudios de caso.

La estructura del artículo parte de una presentación de las variables (condiciones y resultado) que

1 Conclusiones que pueden tener potencial de aplicarse a otros contextos, teniendo en cuenta que la norma generalizada es la existencia de países no homogéneos, que entrarían bajo la denominación de state-nation (Linz y Stepan 2011). van a ser estudiadas. Posteriormente se define en profundidad la variable dependiente y se dan los criterios que determinan su categorización. En el tercer epígrafe se detallan las variables independientes junto con sus categorizaciones. El cuarto apartado presenta y discute los resultados, tras introducir las codificaciones correspondientes en el software de csQCA. El quinto apartado detalla en profundidad las características de la variable que aparece como fundamental: la presencia de conflictos de estatalidad, y el sexto apartado contiene las conclusiones del trabajo.

Por tanto, la pregunta de investigación que orienta el artículo es: ¿qué explica la transición a un sistema democrático en el contexto de la disolución de Yugoslavia?, siendo esencial para su respuesta la comprensión de los conflictos de estatalidad y su naturaleza contingente.

Las unidades de análisis van a ser los territorios que tenían el estatus de repúblicas dentro de la RSFY. Estos territorios son Bosnia-Herzegovina, Croacia, Eslovenia, Macedonia, Montenegro y Serbia. Asimismo, es necesario realizar una acotación temporal, ya que la intención es analizar el comportamiento de los territorios al producirse la disolución de Yugoslavia. Estos procesos iniciados a comienzos de los años 90 no quedaron congelados en el tiempo, pero el objetivo en términos estrictos es considerar el éxito en la transición a un sistema democrático en el momento de la quiebra del régimen autoritario yugoslavo. Dicho marco temporal no supondría sin embargo un obstáculo para el análisis de cambios posteriores, puesto que las variables determinantes del modelo parecen mantener poder explicativo en el tiempo.

La variable dependiente ("resultado" en la lógica QCA) se refiere al éxito o fracaso de cada una de las unidades territoriales a la hora de transitar hacia un régimen democrático. Así pues, el primer paso lógico es la operacionalización del concepto de democracia, cuyos elementos principales (desarrollados con posterioridad) son la existencia de elecciones libres y limpias, la presencia de accountability horizontal y la no limitación de derechos civiles y políticos con posterioridad a las elecciones.

Las variables independientes ("condiciones" en QCA) son el desarrollo económico, la alta heterogeneidad étnica y la presencia de conflictos de estatalidad, mientras que la hipótesis de trabajo se puede formular en los términos siguientes: para que en los países que formaban parte de la antigua Yugoslavia se dé una transición a un sistema democrático, debe haber una conjunción de desarrollo económico, homogeneidad étnica y ausencia de conflictos de estatalidad. Dicho modelo define necesariamente un análisis marcadamente interactivo, para el cual el método QCA resulta especialmente adecuado.

$\mathrm{El}$ acento se pone por tanto en condiciones estructurales previas -en la línea de trabajos como los 
de Lipset (1960) y Bunce (1990) - junto a condiciones internas y el juego de negociaciones y comportamientos por parte de las elites políticas ( $O^{\prime}$ Donnell, Schmitter y Whitehead 1994 y Schmitter 1996).

Para responder a la pregunta de la investigación se va a utilizar el análisis cualitativo comparativo en su variante de crisp-set (en adelante, csQCA) ${ }^{2}$. Este procedimiento permite identificar condiciones causales necesarias, suficientes o las dos anteriores de forma conjunta (Pérez-Liñán 2007). Para ello se utiliza el lenguaje del álgebra booleana, dicotomizando en función de su presencia o ausencia, cada una de las variables incluidas en el modelo ${ }^{3}$. Estaríamos por tanto ante un método cuya lógica subyacente es que una variable no resulta necesaria para la producción de un resultado si aparece como presente y ausente en dos combinaciones que obtienen el resultado positivo (Mahooney 2003).

\section{Definición de las VARIAbles: ¿DE QUÉ dePende LA TRANSICIÓN EXITOSA A LA DEMOCRACIA?}

La ciencia política ha alcanzado gran profundidad conceptual y un amplio conocimiento empírico en el tema que tiene que ver con las transiciones a la democracia, siendo este un destacado tema de estudio en la disciplina. Pese a esto, no hay un acuerdo académico que considere de forma única los factores determinantes en estos procesos transicionales.

Para definir el resultado se va a considerar una definición de democracia procedimental en los términos de Dahl (1971). Los elementos fundamentales van a ser: la existencia de elecciones libres y limpias, tras las cuales no exista un proceso de limitación de derechos políticos para la población, añadiéndose además la presencia de accountability horizontal. La coexistencia de estos tres elementos en cada territorio analizado permitirá considerar la transición como exitosa.

Esta definición no solo considera los tres factores esenciales que Diamond, Linz y Lipset (1990) identificaron en Dahl, sino que además tiene en cuenta el valor de la rendición de cuentas entre poderes del Estado, elemento fundamental, visto el personalismo del régimen anterior y el fortalecimiento de caudillismos regionales por la descentralización del poder (Zaslavsky 1992). Una concepción procedimental de la democracia permite considerar unos mínimos analizables empíricamente, ubicándose además en el terreno de lo que Levitsky llama "expanded procedural mínimum" (Collier y Levitsky 1997: 434). Dicha opción conceptual implica dejar de lado un análisis más amplio o sustantivo de la democracia, optando en su lugar por establecer un umbral observable que atiende además a peculiaridades de la región. Además,

2 Ragin (1987; 2000).

3 Para más información sobre el desarrollo, lenguaje y utilización del método csQCA ver Rihoux y De Mer (2009: 33-68). introducir un alto número de requisitos complejos o que no tienen en cuenta características del entorno, podría producir problemas de falta de variación en la variable dependiente, así como de observación empírica. Teniendo en cuenta el objetivo de este artículo, parece más razonable optar por una estrategia procedimental que mantenga las virtudes señaladas.

En cuanto a las condiciones, el desarrollo económico es un componente clásico en el estudio de las transiciones a la democracia (Lipset 1959 y 1960; Przeworski 1991 y 2000). Un elemento central en su argumentación es que a mayor grado de desarrollo, mayores serán las oportunidades de un territorio para establecer y sostener un régimen democrático (Lipset 1959: 57).

Respecto de la heterogeneidad étnica, para su categorización se van a analizar los censos de población correspondientes a los años 1981 y 1991 (basados en la autoidentificación de los individuos), así como los índices de fragmentación disponibles para el mismo año. La relación teórica esperada es que los países con alta heterogeneidad étnica y cultural tienen mayores dificultades para el planteamiento de objetivos e intereses comunes, los cuales se hacen necesarios en un contexto de cambio político que puede desembocar en una transición democrática.

La presencia de heterogeneidad en términos étnicos no supone de forma necesaria una barrera infranqueable para la transición a un sistema democrático, pero implica una serie de condicionantes en el proceso. Esta relación entre democracia y etnicidad ha sido tratada por autores como Kraus (1996), siendo central la idea de que el pluralismo social que surge en estos casos es complicado de manejar en términos políticos, y puede llevar a enfrentamientos y posturas extremas difícilmente conjugables con un régimen democrático ${ }^{4}$.

La variable referente a la estatalidad aparece recogida por Linz y Stepan (1996) como precondición para la transición y consolidación democráticas, y parece tener especial peso en un contexto marcado por la heterogeneidad etnolingüística. La idea que presentan estos autores es que la democracia se relaciona de forma necesaria con un demos. Si la percepción de quien constituye esa comunidad política no está clara, y además hay fuertes discusiones en torno a quien se considera ciudadano, ello supone un obstáculo que puede resultar insalvable para la transición hacia un sistema democrático.

El conflicto en torno a la estatalidad se traduce en la práctica en la constatación de grandes diferencias en torno a cuáles son los límites territoriales, y sobre quién debe ser ciudadano en la nueva comunidad política (Linz y Stepan 1996: 16). Es importante comprender que dichos conflictos se refieren al compor-

4 En el mismo sentido, ver Collier y Hoeffler (2000: 11) y su referencia a la relación entre la fragmentación étnica y la existencia de conflictos, o la argumentación de Barro (1999: 172) sobre el impacto de la fragmentación en la democratización. 
tamiento de las minorías en el interior de cada república, no siendo una categoría teórica aplicable a los grupos mayoritarios o al comportamiento de cada república respecto de la federación en su conjunto.

\section{TRANSICIÓN A LA DEMOCRACIA Y ACERCAMIENTO METODOLÓGICO: APLICACIÓN DEL MÉTODO QCA}

En este apartado, y atendiendo a la lógica de trabajo que requiere la aplicación del método $\operatorname{csQCA}^{5}$, se va a pasar a la descripción del resultado -transición democrática exitosa- y de las condiciones del modelo -alto desarrollo económico, heterogeneidad étnica y presencia de conflictos de estatalidad-para poder categorizarlas en función de su presencia o ausencia en cada unidad de análisis.

\section{RESULTADO: TRANSICIÓN EXITOSA A UN RÉGIMEN DEMOCRÁTICO}

Todas las repúblicas afrontaron en el momento de la disolución de Yugoslavia un proceso de transición política caracterizado por cambios en la estructura del poder, siendo el rasgo más evidente la convocatoria de elecciones en las que formalmente podría participar más de un partido político. Parecería por tanto que el objetivo de fondo, al menos de cara a la opinión pública, era el de configurar seis sistemas democráticos donde antes existía un único régimen autoritario. Esta idea simplifica la caracterización de los casos negativos de transición exitosa, puesto que formalmente todas ellas inician cambios, pero solo Eslovenia y Macedonia van a cumplir los requisitos para la presencia del resultado. A continuación se van a exponer los argumentos que llevan a considerar a éstos como casos con resultado positivo y a Bosnia-Herzegovina, Croacia, Montenegro y Serbia como casos con resultado negativo.

Eslovenia fue el primer país en legalizar el pluralismo político en marzo de 1990, por lo que ya existían partidos distintos a la Liga Comunista con anterioridad. Esto no implica que el proceso electoral fuese justo per se, pero supone el cumplimiento de una condición necesaria para ello. Fruto de este pluralismo político surge la alianza DEMOS (Demokratična opozicija Slovenije) liderada por el antiguo miembro del partido comunista Jože Pučnik, y que controlaría el Parlamento tras las elecciones.

El otro partido fuerte era el ZKS-SDP, que agrupaba a los miembros del partido comunista, liderados por Milan Kučan, en una nueva formación de carácter democrático. El abanico de opciones políticas no se agotaba con estos dos partidos, ya que existían otras quince agrupaciones registradas en el censo

5 Siguiendo el código de buenas prácticas de Wageman y Schneider (2010). electoral. En total más de 900 candidatos se postularon para su elección en los 16 distritos electorales del país, con una participación que superó el 80 \% en las elecciones parlamentarias (Cohen 1996: 92$3)$. En concurrencia con este proceso también se celebraron elecciones presidenciales, sin ganador en la primera vuelta, pasando a la segunda vuelta los líderes de ZKS-SDP y DEMOS - Jože Pučnik y Milan Kučan. Este último resultó ganador con un 58,3 \% de los votos.

La implantación de un temprano pluralismo político, junto con la ausencia de presiones en el proceso electoral y la variedad de resultados permite hablar de unas elecciones libres y limpias en el caso esloveno, idea que viene apoyada por autores como Aguilera (1994), Cohen (1996), Cohen y Lampe (2011) o Simic (2001).

En cuanto a la limitación posterior de derechos por parte del gobierno esloveno, no se aprecian argumentos histórico-políticos ni bibliográficos que permitan afirmar tal cuestión. La integridad territorial de Eslovenia no sufrió cambios tras las elecciones, y las minorías italianas y húngaras del interior del país siguieron contando con dos escaños reservados y capacidad de veto en los asuntos que afectasen de forma directa sus intereses.

El tercer indicador es la existencia de mecanismos de accountability horizontal. En un sistema de lógica presidencialista como el esloveno, podría resultar preocupante la acumulación excesiva de poder en manos de un presidente que pudiese actuar como un líder autoritario tras su paso por las urnas. Sin embargo los resultados electorales dificultaron la acumulación de poderes, produciendo una cohabitación entre el presidente Kučan -del partido comunista reformado- y el primer ministro Lojze Peterle -de la alianza DEMOS-, ideológicamente ubicada en el centroderecha.

A pesar de su carisma, Kučan siempre se vio obligado a tener en cuenta la actuación del Parlamento, y un hecho esclarecedor fue la iniciativa parlamentaria que hizo dimitir al ministro de defensa, Janez Janša, acusado de traficar con armas para aprovisionar a las Fuerzas de Defensa Territorial Eslovenas ${ }^{6}$. Esta presencia de accountability no tuvo por qué darse de forma necesaria en Eslovenia ni se deriva de un proceso de inevitabilidad histórica. Los resultados de las elecciones y la actuación de las fuerzas políticas en el Parlamento fueron factores decisivos que propiciaron su aparición.

La combinación de un proceso electoral libre y justo, junto con la existencia de accountability horizontal y la ausencia de limitaciones de derechos políticos con posterioridad al proceso electoral y a la formación del Gobierno, permiten categorizar a Eslovenia como caso positivo para el resultado de éxito en la transición a un sistema democrático.

6 Sobre esta cuestión ver Veiga (2011: 59).

RIS [online] 2016, 74 (1), e027. REVISTA INTERNACIONAL DE SOCIOLOGÍA. ISSN-L: 0034-9712 
La república de Macedonia mantuvo sus comicios electorales en octubre de 1990 tras haber legalizado el pluralismo político en abril. Resultado de esta legalización se inscribieron 20 partidos en el registro, siendo los de mayor peso el Movimiento para la Acción Pan-Macedonia (MAAK), el Partido de la Organización Democrática Revolucionaria Interna de Macedonia para la Unidad Nacional de Macedonia (VMRODPMNE) y el Partido para la Prosperidad Democrática (PDPM). Los dos primeros partidos agrupaban los intereses de los ciudadanos de etnia macedonia ${ }^{7}$ mientras que el PDPM agrupaba a los habitantes de etnia albanesa. Junto a estos partidos, construidos sobre un cleavage étnico, estaba el partido de los antiguos comunistas - La Liga de los Comunistas del Partido Macedonio para la Transformación Democrática (SKPDP) - la Alianza de Fuerzas Reformistas (SRJ) y el Partido Socialista (SPM).

La ausencia de mayorías en el Parlamento llevó a un gabinete técnico, presidido por Nikola Kljusev, y en el que tenían representación todos los partidos (Flores 2010: 73). La Presidencia de la República recayó en manos del antiguo líder comunista Kiro Gligorov, elegido por el Parlamento.

Al igual que en el caso de Eslovenia, la presencia del multipartidismo político y la libertad en los medios de comunicación permiten hacer referencia a un caso de elecciones libres y justas, de acuerdo con autores como Aguilera (1994) o Alonso y Maravall (2001).

En cuanto a la restricción de derechos civiles y políticos tras la celebración de elecciones la observación histórica es negativa, al igual que en Eslovenia. El hecho de que el gobierno estuviese formado por una coalición de partidos, y que el presidente Gligorov se atrajese al partido de la minoría albanesa para generar una mayor estabilidad pudieron ser factores clave en este sentido.

El dato que se refiere a la accountability horizontal es más complicado de analizar en el caso macedonio, ya que no existe un acontecimiento que ejemplifique de una forma tan clara su existencia como la dimisión de Janša. A pesar de ello, existen referencias que permiten considerar la moderación en el uso de la autoridad por parte del poder ejecutivo, y el control que ejercía el parlamento sobre él en caso de necesidad (Cohen y Lampe 2011: 67-69).

La combinación de estos tres indicadores permite considerar a Macedonia como el segundo y último caso positivo para la observación del resultado, siendo por tanto Croacia, Montenegro y Serbia los casos con un resultado negativo.

Croacia fue el segundo país en la convocatoria de comicios electorales en la primavera de 1990. La legalización de los partidos políticos había generado un clima en el que agrupaciones antes clandestinas se estaban organizando, estructurando y buscando

7 Formando parte del bloque electoral Frente Nacional Macedonio. fuentes de financiación para concurrir al proceso electoral. Uno de los actores políticos más visibles en aquel momento era la Alianza Democrática Croata (HDZ), que resultaría ganadora de las elecciones con el liderazgo Franjo Tuđman y un marcado discurso nacionalista. A su lado competían más de treinta fuerzas políticas, siendo las alternativas más fuertes el Partido Comunista de Ivica Račan y el partido de centro Coalición para el Acuerdo Nacional (KNS) liderado por Mika Tripalo y Savka Dabčević-Kučar.

Aunque la existencia de varios partidos políticos se confirma para el caso croata, la población serbia fue excluida del proceso electoral, o se encontró con limitaciones muy importantes para participar (Aguilera 1994: 82). La ausencia de elecciones libres y justas bastaría para tratar a Croacia como caso no exitoso en el resultado, pero por el interés que tienen se van a analizar brevemente los restantes indicadores.

La victoria aplastante del HDZ llevó a la presidencia de la república a Tuđman, mientras que el puesto de primer ministro era ocupado por su colega de partido Stjepan Mesić. La deriva autoritaria del Gobierno de Tuđman quedó patente al establecer limitaciones en los derechos cívico-políticos para los habitantes serbios de la región (Aguilera 1994: 82-3 y Cohen 1996: 90). Entre estas medidas se incluían la purga de cargos públicos de origen serbio o el inicio de una fuerte campaña ultranacionalista, proceso en el cual se recuperaron algunos elementos de la simbología "ustacha". En este mismo proceso se encuadra la concentración de poder en manos del presidente, contando con un importante control de manipulación y censura sobre los medios de comunicación, e incluso sobre el poder judicial (Cohen y Lampe 2011: 57).

Puesto que para considerar la existencia de transición a un sistema democrático se debe observar de forma conjunta la presencia de los tres indicadores, Croacia ha de ser considerado como caso negativo ${ }^{8}$. Aunque pudieran plantearse dudas sobre la limpieza del proceso electoral, las limitaciones posteriores de derechos y la ausencia de todo tipo de accountability determinan y refuerzan su categorización negativa.

Bosnia-Herzegovina tuvo sus comicios en noviembre de 1990. Inicialmente hubo un intento de prohibir la formación de partidos con una orientación étnica, pero esta decisión fue invalidada por la Corte Constitucional, surgiendo de hecho un partido político para cada gran grupo étnico en la república serbios, bosniacos y croatas-. Estos eran el Partido Democrático Serbio (SDS) liderado por Rašković, el Partido de la Acción Democrática Musulmana (SDA) de Alija Izetbegović y la Alianza Demócrata Croata (HDZ). Junto a ellos competían dos grandes partidos sin base étnica: el Partido para el Cambio Democrático (SK-SDP) y la Alianza Reformista liderada por Ante Marković. Los resultados de las elecciones parlamentarias llevaron a un complicado sis-

8 En apoyo a esta consideración ver Levitsky y Way (2002). 
tema de gobierno en el que la presidencia era ejercida por Izetbegović, mientras que el primer ministro debía ser croata y el presidente del legislativo de etnia serbia (Cohen 1996: 146).

La situación en Bosnia-Herzegovina difiere radicalmente de las expuestas hasta el momento, y es que a pesar de la existencia de unas elecciones libres y limpias, la fractura en torno a líneas étnicas llevó no solo a la limitación de ciertos derechos civiles y políticos, sino a un estado de guerra -primero civil y luego internacional- en el que se hacía imposible el ejercicio de todo derecho. El estallido bélico del país derrumbó cualquier posibilidad de gobierno democrático, y hasta la paz de Dayton no se sentaron las bases que garantizaran un requisito mínimo como es el control del territorio.

Las dos últimas repúblicas en convocar elecciones fueron Montenegro y Serbia, con comicios electorales en diciembre de 1990. El líder serbio en aquel momento era Slobodan Milošević, que había planteado una modificación de la Constitución para favorecer a su partido y hacer prevalecer el poder del Presidente sobre el Parlamento.

Ante las quejas de los recién nacidos partidos de oposición se llegó a un acuerdo para convocar un referéndum sobre la modificación. La estrategia que siguió Milošević fue la de plantear una consulta acerca de la unidad de Serbia y los derechos de los serbios, de forma que solo incidentalmente se tocaba el tema de la reforma constitucional (Thomas 1999: 70), y consiguiendo así más del $97 \%$ de apoyo a la reforma.

El abanico de partidos que se creó tras la ley de legalización contaba con el Partido Socialista Serbio (SPS) de Milošević, el Movimiento Serbio para la Renovación (SPO) de Vuk Draskovic, el Partido Democrático (DS) de Dragoljub. La Alianza Reformista (SRSJ) de Markovic, junto con el Foro Democrático y el Foro Liberal, representaban las opciones de centro, mientras que en la Vojvodina y Kosovo aparecían partidos con raíces étnicas propias: la Alianza Democrática pro Albanesa de Kosovo y la Unión Democrática de los Húngaros de la Vojvodina.

En las elecciones presidenciales Milošević se impuso al candidato del Movimiento Serbio de Renovación, Drašković, obteniendo más del $65 \%$ de los votos, mientras que el líder del SPO solo consiguió un $16 \%$. Los otros dos candidatos, Ivan Djuric y Sulejman Uglajanin obtendrían poco más del $4 \%$ y el $2 \%$ respectivamente.

Una cuestión importante para la caracterización de las elecciones en Serbia es la que se refiere al pluralismo político. Al contrario que en el resto de las repúblicas, los recién creados partidos de oposición denunciaron a lo largo del proceso irregularidades de todo tipo (Cohen 1996: 153-7 y Thomas 1999: 69-97), llegando incluso a plantear el boicot a las elecciones. Las principales reclamaciones eran el control total de los medios de comunicación por parte del SPS, y el manejo de las reglas electorales de forma que beneficiase directamente los intereses de su partido ${ }^{9}$, poniendo en tela de juicio la justicia y la libertad del proceso electoral. En lo que se refiere a la restricción de derechos tras el proceso electoral, el régimen de Milošević resulta un caso paradigmático. La represión -incluso a través del ejército- de cualquier movilización organizada por la oposición, las constantes acusaciones de corrupción y de fraude electoral, o las actuaciones llevadas a cabo para la represión y el control de la provincia de Kosovo son ejemplos de la misma. Esta reducción del espacio de actuación de la oposición política (Goati 2001) se veía además acompañada por el surgimiento de grupos paramilitares.

Si a este contexto se añade la ausencia de cualquier tipo de rendición de cuentas por parte de Milošević, que desde su elección como presidente fue cambiando de puesto político y dirigiendo el país hasta su caída definitiva en el año 2000, aparece una realidad que difícilmente puede ser considerada como democrática con los parámetros propuestos, por lo que procede su categorización negativa ${ }^{10}$.

Montenegro era una república independiente dentro del sistema federal yugoslavo, pero su situación dio un giro a raíz de los acontecimientos conocidos como la revolución antiburocrática entre 1987 y 1989. Entre julio de 1988 y la primavera de 1989, Milošević organizó más de cien manifestaciones multitudinarias en el territorio serbio y en la vecina república de Montenegro (Thomas 1999: 45). El objetivo de estas movilizaciones era, en última instancia, derrocar los Gobiernos de Vojvodina, Kosovo y Montenegro, sustituyéndolos por otros afines al Gobierno serbio. La estrategia comenzó a dar sus frutos el 5 de octubre de 1988 con la renuncia del Gobierno de la Vojvodina; tan solo dos días después caía el Gobierno de Montenegro y el joven comunista leal a Milošević, Momir Bulatović, accedía al poder, partiendo su agrupación como favorita para los comicios electorales del 9 de diciembre de 1990.

Montenegro comparte algunos problemas con Serbia en relación a la posición de poder del partido mayoritario -SKM-, lo cual impedía la convocatoria de unas elecciones libres y limpias. Aunque se puedan plantear dudas con mayor facilidad que en el caso serbio, el hecho de ser un aliado permanente de la república de Serbia y el haber participado en muchas de sus actuaciones -como las que tienen que ver con la promoción de grupos paramilitares y la limitación de derechos políticos y civiles para determinadas minorías- llevan a considerar el caso de Montenegro como negativo en relación al resultado.

9 Recibiendo menos de la mitad de los votos, el SPS consiguió alcanzar el 77,6 \% de los escaños en la Cámara gracias al sistema electoral planteado en la Constitución hecha por Milošević.

10 En apoyo a esta consideración de Serbia como régimen no democrático en 1990 ver Levitsky y Way (2002) y Goati (2001: 48). 
Si bien no tiene en cuenta exactamente los mismos indicadores, los índices elaborados por Freedom House (Freedom House 1990) vienen a coincidir con estos resultados, dado que los países calificados como no libres para ese año son los mismos que se han categorizado a partir de este análisis. En el mismo sentido se puede mencionar la clasificación de países democráticos llevada a cabo por el proyecto Polity IV (1990), que de igual modo describe como países democráticos a Eslovenia y Macedonia.

\section{Condiciones del MOdelo: desarRollo, hetero- GENEIDAD ÉTNICA Y CONFLICTOS DE ESTATALIDAD}

A través de un esquema similar al empleado para la consideración de los resultados, en este apartado se van a categorizar las condiciones para cada territorio en función de la presencia o ausencia de alto desarrollo económico, de heterogeneidad étnica y de conflictos de estatalidad. Con el objeto de clarificar la propuesta metodológica, resulta necesario hacer referencia a la relación entre las variables y, en concreto, a la distinción conceptual entre heterogeneidad étnica y conflicto de estatalidad.

Mientras que el desarrollo económico se sitúa como una variable diferenciada tanto en términos lógicos como empíricos (no existe un patrón único que lleve a unir el bajo desarrollo económico con los conflictos de estatalidad o con la alta heterogeneidad étnica), esta característica no es inmediatamente predicable de las otras dos condiciones incluidas. A pesar de que conceptualmente parece existir una vinculación entre ellas, la observación empírica permite considerarlas como diferentes a la vista de los resultados. Parecería complicado observar la existencia de un conflicto de estatalidad allí donde la fragmentación etnocultural sea nula, pero no existe correspondencia total entre ambas variables. El conflicto de estatalidad implica un elemento adicional, una activación política, y es por eso que en este trabajo se identifica con la aparición de grupos que cuestionan activamente la configuración de la comunidad política en términos del demos.

El conflicto de estatalidad puede por tanto estar afectado por condicionantes históricos o por otros condicionantes sociopolíticos, pero lo determinante aquí es que no existe un solapamiento total con la presencia de alta fragmentación étnica (ni teórico ni empírico), lo que permite su valoración como diferentes, aunque innegablemente relacionados.

\section{Desarrollo económico}

La dicotomización de esta variable va a tener en cuenta el PIB per cápita de cada república. Para considerar a una república como desarrollada será necesario que esté a una distancia no superior a una desviación tí- pica y media de la media de los países que formaban parte de la OCDE en el año 1990. Esta estimación es coherente en términos generales con la clasificación que ofrece el Banco Mundial, y que considera como países con un nivel de desarrollo alto a aquellos que tengan un ingreso anual per cápita superior a 9.200 dólares (USD) considerando el Producto Nacional Bruto. Siendo la media aritmética de 20.657 USD y la desviación típica de 7.743 , se puede fijar un umbral en torno a los 9.000 USD per cápita, que es superior de hecho al de algunos miembros de la OCDE en aquel año ${ }^{11}$. Los datos económicos de este apartado se encuentran recogidos en las Tablas 1 y 2 .

Tabla 1.

PIB per cápita repúblicas ex yugoslavas y población año 1990

\begin{tabular}{lcc}
\hline Territorio & $\begin{array}{c}\text { PIB per cápita } \\
\text { (USD) }\end{array}$ & $\begin{array}{c}\text { Población } \\
\text { (en miles) }\end{array}$ \\
\hline Bosnia-Herzegovina & 1.800 & 4.516 \\
\hline Croacia & 3.620 & 4.685 \\
\hline Eslovenia & 9.437 & 1.953 \\
\hline Macedonia & 1.470 & 2.131 \\
\hline Montenegro & 3.527 & 644 \\
\hline Serbia & 4.099 & 9.880 \\
\hline
\end{tabular}

Fuente: Kalyvas y Sambanis (2005: 196) y Oficina Estadística de las Naciones Unidas.

Tabla 2.

Producto Nacional Bruto (PNB) en las repúblicas yugoslavas en 1990. USD

\begin{tabular}{ll}
\hline País & PNB \\
\hline Bosnia-Herzegovina & 1.563 \\
\hline Croacia & 3.648 \\
\hline Eslovenia & 8.893 \\
\hline Macedonia & 1.450 \\
\hline Montenegro & 3.705 \\
\hline Serbia & 4.103 \\
\hline
\end{tabular}

Fuente: Banco Mundial.

Eslovenia es por tanto el único país con un alto grado de desarrollo, mientras que Bosnia-Herzegovina, Croacia, Macedonia, Montenegro y Serbia aparecen como repúblicas con bajo nivel de desarrollo (o sin alto desarrollo con la lógica del QCA). A pesar de que Croacia, Serbia y Montenegro presentan en el año 1990 unos niveles elevados en comparación con el resto de países del entorno, ello no es suficiente para considerarlos como países con alto desarrollo en un sentido objetivo.

11 Portugal y Turquía tenían un ingreso per cápita menor a 9000 USD per cápita (7800 y 3400 respectivamente) y Grecia superaba por poco la barrera, al tener un PIB per cápita de 9200 USD. 
Es importante en este punto mencionar por qué no se considera la desigualdad como un dato relevante para considerar el desarrollo. La respuesta viene de la mano del pasado reciente de los territorios analizados, en los cuales la implantación continuada en el tiempo de un sistema comunista había creado unas sociedades relativamente homogéneas, en las que el peso de la desigualdad era menor ${ }^{12}$.

\section{Alta heterogeneidad ÉtNica}

La presencia de alta heterogeneidad étnica se va a considerar positiva cuando el índice de fragmentación étnica ${ }^{13}$ sea superior a 0,42 , decisión que se explica a continuación.

África es la región del mundo con los índices de fragmentación más altos del mundo, existiendo numerosos trabajos que analizan su impacto en el desarrollo económico y político (Easterly y Levine 1997; Barro y Oduro 2002; Fearon 2003). Si se considera el promedio de fragmentación de todos los países del continente africano se obtiene una media de $0,64,{ }^{14}$ con una desviación típica de 0,22. El umbral para la consideración de un alto nivel de fragmentación, que ha sido tasado aquí en un 0,42, es el resultado de restar la desviación típica a la media de la región más fragmentada del mundo. Este umbral resulta además coherente con la bibliografía que estudia el tema de la fragmentación, y en la que países como Botswana (con fragmentación de 0,41 ) es considerado como territorio con una fragmentación sensiblemente menor en la región (Hodler 2006).

Tabla 3.

Índices de fragmentación en las repúblicas yugoslavas.

\begin{tabular}{lc}
\hline País & Fragmentación étnica \\
\hline Bosnia-Herzegovina & 0,6300 (año 1991) \\
\hline Croacia & 0,3690 (año 1991) \\
\hline Eslovenia & 0,2216 (año 1991) \\
\hline Macedonia & 0,5023 (año 1993) \\
\hline Serbia y Montenegro & 0,5736 (año 1991) \\
\hline
\end{tabular}

Fuente: Alesina et al. (2003).

A la vista de los datos presentados en la Tabla 3 se va a considerar como países altamente heterogéneos

12 Milanovic (1998: 15) señala que los niveles relativamente bajos de desigualdad en todos los países de "economía dirigida" no se mantienen igual para Yugoslavia, pero esto es debido a las diferencias entre las repúblicas, no a las desigualdades elevadas entre los ciudadanos que habitaban un mismo territorio.

13 Dicho índice mide la probabilidad de que tomadas al azar dos personas en el territorio de un país, estas dos personas pertenezcan a grupos distintos.

14 Fearon (2003: 23) establece que la media mundial de fragmentación es de 0,47. a Bosnia-Herzegovina, Macedonia, Montenegro y Serbia. Las dos repúblicas noroccidentales -Croacia y Eslovenia- serían las que cumplen los criterios de categorización respecto a los datos censales y del índice de fragmentación, y por tanto se consideran casos negativos de alta fragmentación étnica.

\section{Conflictos en torno a la estatalidad}

La existencia o ausencia de conflictos en torno a la estatalidad es esencial para el trabajo que se está desarrollando. Esta misma importancia fue reconocida por Linz y Stepan (1996), al plantear que un acuerdo sobre la estatalidad es un prerrequisito para la construcción de un sistema democrático. En este trabajo se entiende que existen conflictos de estatalidad cuando en el interior de cada una de las repúblicas surgen movimientos irredentistas, que controlan partes del territorio o que se enfrentan de forma directa la autoridad estatal o incluso la misma idea del Estado tal y como está configurado. Un planteamiento completo de estas cuestiones resultaría en una monografía por derecho propio, por lo que la intención es señalar las líneas básicas de los conflictos y su explicación.

Los movimientos independentistas en BosniaHerzegovina se dejaron sentir desde los primeros compases de la disolución de Yugoslavia, desembocando en una cruenta guerra civil. Los conflictos principales surgieron en las zonas fronterizas con Croacia y Serbia, en las que las minorías étnicas apoyaron movimientos irredentistas. No se trata por tanto de un independentismo que nazca por la imposibilidad de establecer un sistema democrático, sino que se configura como causa y no como efecto de esta imposibilidad.

Croacia encontró un problema similar en la frontera con Bosnia-Herzegovina, en las regiones conocidas como la Krajina y Eslavonia Oriental, habitadas principalmente por población de origen serbio. La intensidad del conflicto crecía a medida que cobraba fuerza el partido nacionalista de Franjo Tuđman, y derivó en incidentes como la rebelión de los troncos.

El caso esloveno es diferente a los demás, ya que la relativa homogeneidad del país y la inclusión de las minorías en la vida política parecían dificultar la aparición de un fenómeno de este tipo. El único conflicto importante registrado en Eslovenia fue el enfrentamiento con el Ejército Popular Yugoslavo tras su declaración de independencia en 1991.

Macedonia es el otro caso que no presenta un conflicto de estatalidad en el interior de sus fronteras. Aunque en esta república la heterogeneidad étnica era elevada, los resultados de las elecciones determinaron la necesidad de un gran gobierno de coalición. Lo que podría haber sido -y de hecho llegó a ser años más tarde- un problema para la de- 
mocracia por falta de gobernabilidad, se convirtió en una herramienta para evitar conflictos en torno a la consideración del demos.

El conflicto en torno a la estatalidad en Serbia comenzó, tras fallecer Tito, con las reivindicaciones de independencia en Kosovo. La población albanesa de Kosovo, que constituía más del $80 \%$ de la población total en la provincia, había iniciado una serie de movimientos que buscaban desde una mayor autonomía hasta la independencia, siendo común a todos ellos el cuestionamiento de quién formaba el demos político.

La intervención directa de Milošević en el conflicto -en 1989 con los acontecimientos de Gazimestán- supuso un recrudecimiento de las tensiones. El conflicto en torno a la estatalidad se hizo más fuerte, y se convirtió en una importante baza política para Milošević y el SPS. En este camino surgieron o se intensificaron los enfrentamientos con grupos guerrilleros, incluso se produjo el establecimiento de un sistema público paralelo en Kosovo, a través del cual los albaneses tenían, informalmente, sus propias escuelas, hospitales, y cauces administrativos (Judah 2002: 61-98).

Montenegro supone un caso especial en cuanto a la determinación del conflicto de estatalidad, por cuanto la naturaleza del mismo afectó en un doble sentido a la pequeña república. Por un lado la determinación del demos en su relación con Serbia, y por otro su posición hacia Kosovo. Respecto al primer asunto, la llamada revolución antiburocrática encabezada por partidarios de Milošević había puesto en el gobierno a un partidario claro de Serbia, Bulatović. Siendo esto así, la primera dificultad que debía afrontar Montenegro en relación con la estatalidad era la determinación o no de su identidad junto con Serbia. Dicha cuestión se resolvió con relativa calma por la cercanía de sus líderes, dando lugar a un Montenegro que apoyó a Serbia durante la década de los 90 . El alineamiento montenegrino con Serbia le llevó a ser una parte activa en el conflicto de Kosovo, asunto que se veía ade- más alentado por la presencia de una minoría albanesa en Montenegro. Con esta descripción de los acontecimientos Eslovenia y Macedonia serían las repúblicas donde no aparecen conflictos en torno a la estatalidad, mientras que Bosnia-Herzegovina, Croacia, Montenegro y Serbia sí presentan conflictos de esta naturaleza.

A modo de anexo, la relación de la concentración territorial de los grupos con la posibilidad de insurgencia ha sido analizada por Gurr (1993), y ayuda a comprender en qué circunstancias la rebelión es una alternativa más probable en los casos analizados. Sin embargo este elemento no guarda relación directa con el resultado ni se solapa de forma perfecta con los conflictos de estatalidad. Su estudio en detalle tendría mejor encaje conceptual en un trabajo que pretendiese desentrañar los condicionantes de los conflictos de estatalidad (en lugar de analizar su impacto concreto en las transiciones) y es por ello que no se integra como condición formal del modelo.

\section{AnÁlisis csQCA}

El primer paso para la aplicación del método es la construcción de una tabla de la verdad, en la que se presente la dicotomización de cada una de las variables (ver Tabla 4). Lo que se busca con esta tabla es presentar la información condensada, de forma que cada variable aparezca categorizada en función de su presencia o ausencia. Los resultados del análisis aparecen en los Gráficos 1, 2 y 3, que recogen las soluciones compleja, simple e intermedia respectivamente ${ }^{15}$.

15 En la lógica de análisis QCA * significa adición y el símbolo - negación de la condición que aparece inmediatamente después. En este caso la primera combinación (desarrollo*heterogeneidad*-estatalidad) se leería: desarrollo económico más ausencia de heterogeneidad más ausencia de conflicto estatalidad.

Tabla 4.

Tabla con los resultados para el análisis QCA.

\begin{tabular}{lcccc}
\hline Países & $\begin{array}{c}\text { Exito en la transición } \\
\text { democrática }\end{array}$ & $\begin{array}{c}\text { Alto Desarrollo } \\
\text { Económico }\end{array}$ & $\begin{array}{c}\text { Heterogeneidad } \\
\text { étnica }\end{array}$ & $\begin{array}{c}\text { Conflictos de } \\
\text { estatalidad }\end{array}$ \\
\hline Bosnia-Herzegovina & No & No & Sí & Sí \\
\hline Croacia & No & No & No & Sí \\
\hline Eslovenia & Sí & Sí & No & No \\
\hline Macedonia & Sí & No & Sí & No \\
\hline Montenegro & No & No & Sí & Sí \\
\hline Serbia & No & No & Sí & Sí \\
\hline
\end{tabular}

Fuente: Elaboración propia. 
Gráfico 1.

Resultados QCA (solución compleja) ${ }^{16}$.

\begin{tabular}{lccc}
\hline & Raw coverage & Unique coverage & Consistency \\
\hline desarrollo*-heterogeneidad*-estatalidad & $0.500-000$ & 0.500000 & 1.00000 \\
\hline -desarrollo*heterogeneidad*-estatalidad & 0.500000 & 0.500000 & 1.00000 \\
\hline Solution coverage & 1.00000 & & \\
\hline Solution Consistency & 1.00000 & & \\
\hline
\end{tabular}

Gráfico 2.

Resultados QCA (solución parsimoniosa) ${ }^{17}$.

\begin{tabular}{lccc}
\hline & Raw coverage & Unique coverage & Consistency \\
\hline -estatalidad & 1.00000 & 1.00000 & 1.00000 \\
\hline Solution coverage & 1.00000 & & \\
\hline Solution Consistency & 1.00000 & & \\
\hline
\end{tabular}

Gráfico 3.

Resultados QCA (solución intermedia) ${ }^{18}$.

\begin{tabular}{lccc}
\hline & Raw coverage & Unique coverage & Consistency \\
\hline -estatalidad & 1.00000 & 1.00000 & 1.00000 \\
\hline Solution coverage & 1.00000 & & \\
\hline Solution Consistency & 1.00000 & & \\
\hline
\end{tabular}

La solución compleja supone optar por el resultado más conservador de los disponibles, que será tomado como referencia para el análisis posterior suponiendo un riesgo menor, dado que no asume un valor predeterminado para las configuraciones causales sin observación. Pese a ello, también se incluyen las soluciones intermedia y parsimoniosa en los gráficos 2 y 3 .

En el gráfico de solución compleja se puede ver cómo las dos configuraciones que producen transición a un sistema democrático son: la conjunción de desarrollo económico, con ausencia de alta heterogeneidad y ausencia de conflictos de estatalidad (Eslovenia) y la conjunción de ausencia de alto desarrollo económico con presencia de alta heterogeneidad étnica y ausencia de conflictos en torno a la estatalidad (Macedonia) ${ }^{19}$. Los indicadores de raw

16 Toma todos los contrafácticos (combinaciones posibles por las condiciones introducidas que no tienen observación empírica) como falsos, de ahí la mayor complejidad de las combinaciones.

17 Contrafácticos tomados como verdaderos, lo que permite obtener una solución más parsimoniosa.

18 Contrafácticos tomados en función de la relación teórica esperada de cada variable con el resultado. Para democracia sería desarrollo presente, heterogeneidad ausente y estatalidad ausente.

19 El símbolo * marcaría la unión de elementos, siendo el equivalente en álgebra booleana a la conjunción "y". El símbolo se refiere a la ausencia de la variable a la que precede, coverage, unique coverage y consistency señalan que no existe ningún caso observado en el que una de esas dos configuraciones causales lleve a un resultado negativo para la variable dependiente, siendo capaces de explicar además la totalidad de los casos positivos observados. Especialmente valioso para el análisis es el nivel consistencia, que podría generar problemas de interpretación en situaciones de inconsistencia con un número de casos reducido.

A la vista de estos datos parecería que ni el desarrollo económico ni la cuestión de la heterogeneidad cultural fueron factores que determinasen el éxito o el fracaso en la transición a un sistema democrático (aparecen como presentes y ausentes en las dos configuraciones con resultado positivo). La simplificación de las fórmulas causales que llevan a un valor positivo para el resultado permite tratar como condición suficiente la ausencia de conflictos en torno a la estatalidad. Esta conclusión apoya la tesis de que en momentos de democratización pueden existir herramientas que eviten el choque de identidades etnonacionales (Jahn 1992).

Siendo esta condición el elemento determinante, es pertinente realizar un desarrollo en profundidad de la misma, explicando su aparición y su comportamiento en cada uno de los territorios.

$\overline{\text { siendo por tanto el }}$ operador lógico que en álgebra booleana indica negación. 


\section{APARICIÓN Y RELEVANCIA PRÁCTICA DE LOS CONFLICTOS DE ESTATALIDAD}

El análisis con csQCA permite observar cómo la condición suficiente para el éxito en la transición a un sistema democrático es la ausencia de conflictos en torno a la estatalidad. La fuerza de esta condición se presenta en un doble sentido, ya que, por el lado de los casos exitosos, la única nota común entre Eslovenia y Macedonia es la ausencia de conflictos de estatalidad y, por el lado de los casos no exitosos, es el factor que diferencia a Croacia de Eslovenia por un lado, y a Macedonia de Bosnia-Herzegovina, Montenegro y Serbia por otro.

Un aspecto que debe ser considerado en esta línea de argumentación es la dirección de la causalidad que, en la discusión planteada, va desde la aparición de conflictos de estatalidad (causa) al fracaso en la transición a un régimen democrático (efecto). Para desentrañar esta relación el mejor instrumento es la observación histórica de los acontecimientos, que permite dar cuenta del orden lógico de los mismos y ofrece indicios sólidos para su interpretación en el sentido antes apuntado. Si esta interpretación es correcta se deberán apreciar las causas con anterioridad al efecto, esto es, la falta de acuerdo en torno al demos debe preceder a la celebración de las elecciones (que a su vez anteceden lógicamente a la limitación de derechos políticos y a la ausencia de accountability). En todos los casos que presentan un resultado negativo para la variable dependiente el cuestionamiento de la comunidad política es un paso previo a los comicios electorales, lo cual no es obstáculo para que tras la celebración de los mismos se produzcan tensiones que refuercen ambos fenómenos, dada la relación fluida que existe entre ellos. El caso más claro que apunta a esta precedencia de los conflictos de estatalidad es el que se refiere a la república Serbia, en el que las tensiones y desacuerdos territoriales aparecen incluso antes del proceso de disolución de Yugoslavia. Teniendo en cuenta que los conflictos de estatalidad en otras repúblicas tuvieron a la minoría serbia como uno de los actores principales, parece razonable defender la dirección causal en los términos planteados. Además, se puede observar cómo la desaparición de los conflictos de estatalidad es un catalizador para las transiciones a la democracia, como se apuntará posteriormente en este apartado.

Fuese o no el deseo de las elites políticas en Eslovenia y Macedonia, los resultados electorales favorecieron la unión entre fuerzas políticas. En Eslovenia la coalición DEMOS agrupaba a más de seis fuerzas políticas, mientras que en Macedonia se hizo necesario un gobierno de coalición con representación albanesa y macedonia. El reparto de cuotas de poder entre los diferentes grupos étnicos funcionó como mecanismo de estabilidad, encauzando la transición hacia modelos democráticos, y aplacando consignas nacionalistas excluyentes.
En Serbia la situación había transcurrido por caminos muy diferentes, siendo la exaltación del nacionalismo un arma política de uso frecuente. La llegada al poder de Slobodan Milošević estuvo caracterizada por su presentación como defensor de los serbios (Thomas 1999 o Veiga 2004). De esta afirmación no se desprende en modo alguno que Milošević fuese el creador del nacionalismo serbio, ya que dicho nacionalismo existía, y tenía un reflejo en la composición social del territorio y en la obra de algunos intelectuales. El paso que dio Milošević fue el de convertir de nuevo esta identidad en una herramienta política, construyendo sobre ella una base de su poder. En una época marcada por la crisis económica y política, fue capaz de canalizar el descontento de la población serbia, y lo hizo a través de un planteamiento radical que alentó el conflicto de estatalidad.

El proceso de control por parte de Milošević se extendió a Montenegro a partir de la revolución antiburocrática. A partir de ese momento la influencia serbia en la república fue total, hasta el punto de extender el conflicto de estatalidad al territorio montenegrino, en el cual se movilizaban tropas de policía para la provincia y se llevaban a cabo manifestaciones a favor de un Kosovo serbio casi con la misma regularidad que en Belgrado ${ }^{20}$. Este curso de actuaciones tuvo su importancia respecto a los conflictos de estatalidad en un doble sentido. Por un lado, el renacimiento del nacionalismo serbio tuvo sus efectos en otros territorios yugoslavos en los que había importantes concentraciones de serbios -Bosnia-Herzegovina y Croacia- y por otro lado fortaleció al nacionalismo albanés en su propio territorio (Kosovo).

En el territorio croata el conflicto en torno a la estatalidad surge en un contexto en el que no existía una alta fragmentación étnica; a este respecto es importante señalar el papel de dos grandes figuras políticas en la región, Franjo Tuđman y Slobodan Milošević.

El camino al poder para Tuđman estuvo marcado por la utilización de símbolos nacionalistas croatas. Su utilización se llevó hasta el extremo, apareciendo discursos a favor de los croatas como único pueblo constituyente de la república, y negando derechos políticos a ciudadanos de otras etnias, especialmente a los serbios. Los ciudadanos serbios en Croacia asistían a un doble proceso político, que se manifestaba en la amenaza de un nacionalismo excluyente en el interior de sus fronteras, y en la existencia de apoyos en Belgrado. Viendo la polarización entre ambos procesos no resulta extraño el surgimiento de un conflicto en torno a la estatalidad, alentado por dos discursos políticos encontrados. El caso croata muestra la aparición de un conflicto en un contexto en el que formalmente no hay una alta fragmentación étnica, y señala de forma especial a la importancia de las actuaciones políticas en los procesos que se analizan.

20 Para más información sobre este proceso consultar Thomas (1999: 44-51). 
Los acontecimientos en Bosnia-Herzegovina fueron especialmente clarificadores para la defensa del argumento que se presenta. Aunque tras la legalización de partidos políticos la línea de competición se articuló en torno a líneas étnicas, esta se mantuvo dentro de márgenes pacíficos hasta después de las elecciones. A medida que la tensión aumentaba en los países vecinos y se arrinconaban los elementos moderados, también se iba complicando la situación de Bosnia-Herzegovina.

La victoria electoral del HDZ de Tuđman y del SPS en Serbia fortaleció a las ramas de ambos partidos en Bosnia, que comenzaron a alentar un discurso radical en torno al cual se fortaleció el conflicto de estatalidad. No es que la comunidad musulmana fuese un mero espectador en este proceso, pero la selección del moderado Izetbegovic como presidente de la república contribuyó a calmar temporalmente los ánimos, hasta su desbordamiento definitivo con el inicio de una guerra abierta.

Continuando con la argumentación de la importancia de los conflictos de estatalidad, parece pertinente reseñar brevemente la relación existente entre su superación y el inicio de procesos de democratización, relación que parecería la esperada de confirmarse su carácter de condición suficiente para los casos analizados.

En Bosnia-Herzegovina la superación -no necesariamente definitiva- de los conflictos en torno a la idea de estatalidad aparece en torno al año 2005, fecha en la que se levanta la tutela internacional sobre el país. La nueva organización territorial, que se articula en torno a un modelo confederal con dos repúblicas -Herzegovina y la República Srpska- supone un arreglo institucional que ha permitido lidiar con los problemas de estatalidad, alumbrando un proceso de cambio y transición. El índice de Freedom House recoge este cambio, y considera a Bosnia-Herzegovina como país parcialmente libre desde el año 2006.

En Croacia el fallecimiento de Tuđman en 1999 supuso un cambio en las filas del HDZ, que se acercó a líneas más moderadas, cambiando el discurso nacionalista radical. La migración -voluntaria y forzosa- de serbios de la Krajina y de Eslavonia Oriental debilitó la estructura básica de aquellos que mantenían un pulso sobre la estatalidad en Croacia, consiguiendo un efecto importante que acompañaba a la moderación dentro del HDZ, tal y como se recoge en los índices de Polity IV para ese año.

Macedonia es un caso importante para este estudio porque el proceso que se observa llevaba un camino inverso al de Bosnia-Herzegovina o Croacia. La intervención de la OTAN en Kosovo produjo un importante flujo migratorio de albaneses hacia Macedonia, y junto a ellos, de las guerrillas que habían estado operando en territorio kosovar. A principios del año 2001 la aparición de estos conflictos pudo desembocar en un problema de estatalidad para Macedonia, que sin embargo fue capaz de en- cauzarlos a través de los acuerdos de Ohrid y de la mediación internacional, con los cuales se estableció un panorama político más equilibrado, en el que la minoría albanesa aceptaba de forma definitiva los arreglos institucionales y la constitución del país.

Serbia y Montenegro, que mantuvieron una unión política a partir del año 1992 también tuvieron un proceso similar al de Croacia. La caída de Slobodan Milošević, desprestigiado a nivel internacional e interno por la guerra y sus atrocidades, supuso un punto de inflexión en la política del país. Al desaparecer el artífice político de importantes movilizaciones nacionalistas radicales, y agotada la idea de una Gran Serbia en la región, fue posible una transición a la democracia.

Aunque no se puede ignorar la posible influencia de otras variables no contempladas en el modelo, y que pudieron afectar a los procesos de transición en épocas posteriores, parece confirmarse la importancia de los problemas de estatalidad en la región y su relación con la democracia.

El punto que se ha querido transmitir en este desarrollo es la relevancia de las actuaciones políticas para la aparición de conflictos en torno a la estatalidad. Ni surgen inevitablemente en un país altamente heterogéneo en el que incluso hay concentración territorial de los diferentes grupos étnicos (Macedonia) ni resultan imposibles en ausencia de esta fuerte heterogeneidad étnica (Croacia). Además su naturaleza es la de un fenómeno esencialmente dinámico, que cambia en función de las coyunturas políticas y principalmente con las actuaciones de los actores políticos.

\section{Conclusiones}

A través de la aplicación de un análisis cualitativo comparativo, se ha llegado a un resultado que pone de relieve la importancia de los conflictos de estatalidad en los procesos transicionales en Yugoslavia. La ausencia del mismo lleva a la transición exitosa para los casos estudiados, mientras que su presencia imposibilita dicho resultado.

El conflicto de estatalidad se relaciona con el nacionalismo y las identidades, pero tiene un añadido fundamental relacionado con las actuaciones políticas que rodean su aparición. Como se ha señalado con anterioridad, la alta fragmentación étnica en un territorio no garantiza la aparición de dicho fenómeno, y por tanto no sería exacta la generalización convencional que une los diferentes caminos de las repúblicas ex yugoslavas con la presencia de mayor o menor heterogeneidad dentro de sus fronteras. La naturaleza contingente del conflicto de estatalidad se dispone como otro elemento fundamental en las conclusiones del trabajo.

Por otro lado, la falta de relevancia del desarrollo económico en estos procesos debe ser tomada con 
cautela, dado que solo existe una observación positiva para dicha condición (Eslovenia), lo que impide obtener resultados plenamente sólidos en la cuestión. Sería interesante por tanto evaluar el papel del desarrollo en conjunción con las otras condiciones contempladas en el modelo en contextos más amplios, que permitan mayor número de configuraciones cubiertas por observación empírica. En cualquier caso el hecho de que esta condición no aparezca como suficiente o necesaria en el análisis no supone en absoluto que no tenga importancia, o que deba obviarse en otros estudios de la cuestión.

Como señala Snyder (2000), existe la posibilidad de que en contextos de democratización las elites políticas opten por alentar movimientos nacionalistas afines a su causa, como medio para permanecer en el poder. El efecto directo de estas políticas puede ser precisamente la imposibilidad de continuar con los procesos transicionales, lo que se puede observar de forma paradigmática en el caso serbio. Ante un proceso de cambio y de incertidumbre, el fortalecimiento de un discurso nacionalista actúa como elemento aglutinador, en torno al cual se puede agrupar una fuerza capaz de frenar una corriente democratizadora o demandas sociales en ese sentido.

La aplicación del método QCA se ha revelado como una herramienta de gran utilidad para la comprensión de las transiciones en las ex repúblicas yugoslavas, puesto que permite aplicar una lógica comparada y multicausal al fenómeno objeto de estudio. Además, y dada la naturaleza del fenómeno, permite lidiar con la problemática del reducido número de casos e introducir un análisis novedoso en el estudio de un acontecimiento sociopolítico de índole histórica.

Estas observaciones abren un interesante terreno de investigación en la línea de las democratizaciones en contextos multinacionales y étnicamente diversos. En estas situaciones los conflictos de estatalidad pueden desplegar todo su potencial para dificultar las transiciones, siendo esenciales las capacidades personales e institucionales para mantener los conflictos bajo control. Sería por tanto de gran interés analizar el comportamiento de esta variable en otros contextos geográficos, que compartan el potencial para la aparición de conflictos de estatalidad, considerando además el dinamismo del fenómeno y su posible su expresión o no a lo largo del tiempo.

\section{Agradecimientos}

Quisiera agradecer a Iván Llamazares Valduvieco por sus valiosas y pertinentes aportaciones y por su tiempo en los intercambios imprescindibles para la elaboración de este artículo, así como a Manuel Alcántara, Fátima García y Salvador Martí por sus comentarios sobre una versión preliminar del mismo. También agradecer a los dos evaluadores anónimos de la RIS por su participación en la mejora de este trabajo.

\section{Referencias Bibliográficas}

Aguilera, C. 1994. "Los nacionalismos en la desintegración de Yugoslavia". Revista CIDOB $d^{\prime}$ 'afers internacionals 27:77-93.

Alesina, A., A. Devleeschauwer, W. Easterly, S. Kurlat and R. Wacziarg. 2003. "Fractionalization". Journal of Economic Growth 8:155-194. http://dx.doi. org/10.1023/A:1024471506938

Alonso, S. y J. M. Maravall. 2001. "Democratización en la periferia europea". Revista Española de Ciencia Política 5:7-47.

Barro, A. and A. Oduro. 2002. "Ethnic fractionalization in African labour market". Journal of Development Economics 68:355-379. http://dx.doi.org/10.1016/ S0304-3878(02)00017-2

Barro, R. 1999. "Determinants of democracy". Journal of Political Economy 107:158-183. http://dx.doi. org/10.1086/250107

Bunce, V. 1990. "The Struggle for Liberal Democracy in Eastern Europe". World Policy Journal 7:395-430.

Cohen, L. J. 1996. Broken bonds: Yugoslavia's Disintegration and Balkan Politics in Transition. Boulder: Westview Press.

Cohen, L. and J. Lampe. 2011. Embracing democracy in the western Balkans. From post conflict struggles toward European integration. Pennsylvania, NW: Woodrow Wilson Centre Press.

Collier, P. and A. Hoeffler. 2000. Greed and grievance in civil war. Washington, DC: World Bank Development Research Group.

Dahl, R. 1971. Polyarchy: participation and opposition. New Haven: Yale University Press.

Diamond, L. 1990. "Nigeria: Pluralism, Statism and the Struggle for Democracy". Pp. 351- 409 en Politics in developing countries. Comparing experiences with democracy, edited by L. Diamond et al. Boulder, CO: Lynne Rienner.

Diamond, L. J., J. Linz and S. M. Lipset. 1990. Politics in developing countries. Comparing experiences with democracy. Boulder, CO: Lynne Rienner.

Easterly, W. and R. Levine. 1997. "Africa's Growth Tragedy: Policies and Ethnic Divisions". The Quarterly Journal of Economics 113:1203-1249. http:// dx.doi.org/10.1162/003355300555466

Fearon, J. 2003. "Ethnic and cultural diversity by country". Journal of Economic Growth 8:195-222. http:// dx.doi.org/10.1023/A:1024419522867

Flores, C. 2010. "Los acuerdos de paz de Dayton, el acuerdo marco de Ohrid y la cuestión del estatuto final de Kosovo". Balkania: Revista de Estudios Balcánicos 1:28-47.

Glenny, M. 1996. The Fall of Yugoslavia: The third Balkan war. New York: Penguin Books.

Goati, V. 2001. "The Nature of the Order and the October Overthrow in Serbia". Pp. 45-58 in Revolution and Order: Serbia after October 2000, edited by I. Spasic and M. Subotic, Belgrado: Institute for Philosophy and Social Theory.

Gurr, T. 1993. "Why Minorities Rebel: A Cross National Analysis of Communal Mobilization and Conflict since 1945". International Political Science Review 14:161-201. http://dx.doi. org/10.1177/019251219301400203

Hodler, R. 2006. "The curse of natural resources in fractionalized countries". European Economic Review 
50:1367-86. http://dx.doi.org/10.1016/j.euroecorev.2005.05.004

Janos, A. 1997. Czechoslovakia and Yugoslavia. Ethnic conflict and the dissolution of multinational states. International and Area Studies: University of California at Berkeley.

Judah, T. 2000. Kosovo: war and revenge. New Haven: Yale University Press.

Kalyvas, S. and N. Sambanis. 2005. "Bosnia's Civil War: Origins and Violence Dynamics". Pp. 191-230 in Understanding Civil War, vol. 2, Europe, Central Asia, and Other Regions: Evidence and Analysis, edited by P. Collier and Nicholas Sambanis. Washington: World Bank Publications.

Kraus, P. 1996. "Problemas de democratización en los Estados plurinacionales". Revista Internacional de Filosofía Política 8:59-80.

Levitsky, S. and L. Way .2002. "The rise of competitive authoritarianism". Journal of Democracy 13:51-65. http://dx.doi.org/10.1353/jod.2002.0026

Linz, J. and A. Stepan. 1996. Problems of democratic transition and consolidation. Baltimore: The John Hopkins University Press.

Linz, J. and A. Stepan. 2001. "Political Identities and electoral sequences: Spain, The Soviet Union and Yugoslavia". Pp. 200-212 in Arguing Comparative Politics, edited by A. Stepan. Oxford University Press.

Lipset, S. M. 1959. "Some Social Requisites of Democracy: Economic Development and Political Legitimacy". American Political Science Review 53:69-105. http://dx.doi.org/10.2307/1951731

Lipset, S. M. 1960. Political man; the social bases of politics. Garden City, N.Y.: Doubleday.

Milanovic, B. 1998. Income, Inequality and Poverty during the Transition from Planned to Market Economy. Washington D.C.: The World Bank.

O'Donnell, G. P. Schmitter y L. Whitehead. 1994. Transiciones desde un gobierno autoritario. Barcelona: Paidós.

Palacios, J. M. 2003. Transición democrática poscomunista. Democratización y estatalidad en la Unión Soviética y en Yugoslavia. Madrid: Ediciones UNED.

Pérez-Liñán,A. 2007. "El Método Comparativo: Fundamentos y Desarrollos Recientes". Documento de trabajo: Universidad de Pittsburgh, Pittsburgh, PA.

Przeworski, A. 1991. Democracy and the market: political and economic reforms in Eastern Europe and Latin America. Cambridge: Cambridge University Press. http://dx.doi.org/10.1017/CBO9781139172493

Przeworski, A. 2000. Democracy and development: political institutions and well-being in the world, 19501990. Cambridge: Cambridge University Press. http://dx.doi.org/10.1017/CBO9780511804946

Przeworski, A. and F. Limongi. 1997. "Modernization: theories and facts". World Politics 49:155-183. http:// dx.doi.org/10.1353/wp.1997.0004
Ragin, C. 1987. The comparative method: moving beyond qualitative and quantitative strategies. Berkeley: University of California Press.

Ragin, C. 2000. Fuzzy-set social science. Chicago: University of Chicago Press.

Rihoux, B. and G. De Mer. 2009. «Crisp-Set Qualitative Comparative Analysis (csQCA)». Pp. 3369 in Configurational Comparative Methods. Qualitative Comparative Analysis (QCA) and Related Techniques, edited by B. Rihoux and C. Ragin. Thousand Oaks: Sage. http://dx.doi. org/10.4135/9781452226569.n3

Schmitter, P. 1996. "The influence of the international context upon choice of national institutions and policies in neo-democracies". Pp. 33-69 in The International Dimensions of Democratization: Europe and the Americas, edited by L. Whitehead. Oxford University Press.

Schneider, C. and C. Wagemann. 2010. "Standards of Good Practice in Qualitative Comparative Analysis (QCA) and Fuzzy-Sets". Comparative Sociology 9:397-418. http://dx.doi.org/10.1163/15 6913210X12493538729793

Silber, L. and A. Litle. 1997. The Dead of Yugoslavia. Penguin Books.

Simic, P. 2001. "Yugoslavia en la encrucijada ¿reformas o desintegración?". ISTOR II:51-70.

Smith, A. y R. Máiz. 2003. Nacionalismos y movilización política. Buenos Aires: Prometeo Libros.

Smith, A. 1991. National identity. Reno: University of Nevada Press.

Snyder, J. 2000. From voting to violence: democratization and nationalist conflict. New York: Norton.

Taibo, C. 1998. Las transiciones en la Europa Central y Oriental. ¿Copias de papel carbón?. Madrid: Los libros de la Catarata.

Thomas, R. 1999. Serbia under Milošević. Politics in the 1990s. London: Hurst and Company.

Trbovich, A. 2008. A legal geography of Yugoslavia's disintegration. New York: Oxford University Press. http://dx.doi.org/10.1093/acprof:oso/9780195333 435.001 .0001

Veiga, F. 1994. La trampa balcánica. Barcelona: Grijalbo.

Veiga, F. 2004. Slobo, una biografía no autorizada de Slobodan Milošević. Barcelona: Debate.

Veiga, F. 2011. La fábrica de las fronteras. Guerras de secesión yugoslavas 1991-2001. Madrid: Alianza Editorial.

Zaslavsky, V. 1992. "Nationalism and Democratic Transition in Post-communist Societies". Dedalus 121:97-121.

HUGO MARCOS-MARNÉ es investigador y candidato a doctor en el área de Ciencia Política de la Universidad de Salamanca. Sus principales líneas de investigación tienen que ver con la política comparada y las identidades nacionales, temas sobre los que ha publicado trabajos en la Revista Española de Ciencia Política, Revista de Estudios Políticos o National Identities entre otras. 